Research Article

\title{
Study on Single-Machine Group Scheduling with Due-Window Assignment and Position-Dependent Weights
}

\author{
Weiguo Liu (iD, Xuyin Wang, Xiaoxiao Wang, and Peizhen Zhao
}

Business School, Northwest Normal University, Lanzhou 730070, China

Correspondence should be addressed to Weiguo Liu; lwgwinterplum@163.com

Received 24 May 2021; Revised 3 August 2021; Accepted 11 August 2021; Published 26 August 2021

Academic Editor: Samuel Yousefi

Copyright (C) 2021 Weiguo Liu et al. This is an open access article distributed under the Creative Commons Attribution License, which permits unrestricted use, distribution, and reproduction in any medium, provided the original work is properly cited.

This article considers a single-machine group scheduling problem with due-window assignment, where the jobs are classified into groups and the jobs in the same group must be processed in succession. The goal is to minimize the weighted sum of lateness and due-window assignment cost, where the weights depend on the position in which a job is scheduled (i.e., position-dependent weights). For the common, slack, and different due-window assignment methods, we prove that the problem can be solved polynomially, i.e., in $O(N \log N)$ time, where $N$ is the number of jobs.

\section{Introduction}

Group scheduling (GS) is an approach, which schedules jobs with similar characteristics close together and reduces tooling changeovers and in-process inventories to improve efficiency in high-volume production (Neufeld et al. [1], Lu et al. [2], Yin et al. [3], and Wang and Liu [4]). Wang and Wang [5] studied the single-machine group scheduling problem with ready times and time-dependent processing times. Under the case of the group setup times and job processing times are proportional linear deterioration functions, they proved that the makespan minimization problem can be solved in polynomial time. He and Sun [6] considered the single-machine group scheduling problem with general deteriorating jobs and learning effects. They proved that the makespan and the sum of completion times minimizations remain solvable in polynomial time. $\mathrm{Lu}$ et al. [7] considered the single-machine group scheduling with learning effects and resource allocation. For the makespan minimization subject to limited resource availability, they proved that the problem can be solved in polynomial time under some special cases. For the general case, they proposed heuristic and branch-and-bound algorithms. Zhang et al. [8] studied the single-machine group scheduling with positiondependent processing times. For the makespan and the total completion time minimizations, they proved that the problem can be solved in polynomial time. Liu et al. [9] considered the single-machine group scheduling with the proportional deterioration effect. For the makespan minimization with release times, they proposed heuristic and branch-and-bound algorithms. Huang [10] studied the single-machine group problem with proportional deterioration effects, where the total weighted completion time minimization is the primary criterion and the maximum cost minimization is the secondary criterion. They proved that the problem remains solvable in polynomial time. Wang and Liang [11] and Liang et al. [12] considered the singlemachine group scheduling problem with deteriorating jobs and resource allocation. Qin et al. [13] considered the flowshop group scheduling problem with learning effects. For some regular objectives (including the makespan, total completion time, total weighted completion time, and maximum lateness), they proposed heuristics and metaheuristics.

On the other hand, due to the increasing interest in the just-in-time (JIT) system, the problem of the due-date assignment has been closely focused on by scholars (Yin et al. [14], Wang et al. [15], and Shabtay [16]). However, under the group technology, there are relatively few studies on the problem of the assignment of jobs. Li et al. [17] studied the single-machine group scheduling with due-date assignment. For the common (CON), slack (SLK), and different (DIF) due-date assignment methods, they proved 
that the nonregular objective minimization can be solved in polynomial time. Sun et al. [18], Lv et al. [19], and Wang et al. [20] considered single-machine group scheduling problems with resource allocation and learning effect. Under slack due-date assignment and the linear and convex resource consumption functions, Sun et al. [18] gave some results. Lv et al. [19] showed by two counter examples that the results of Sun et al. [18] were incorrect. Under the convex resource allocation, for some special cases, Wang et al. [20] proved that the problem can be solved in polynomial time. For the general case of the problem, Wang et al. [20] proposed the heuristic, tabu search, and branch-and-bound algorithms.

Recently, Wang et al. [21] considered the single-machine group scheduling problem with due-date assignment and positional-dependent weights. For the CON, SLK, and DIF due-date assignments, they proved that the problem can be solved in polynomial time. The importance of duewindow assignment scheduling is widely recognized for the production company [22-27]. Ji et al. [28] investigated the single-machine group scheduling with slack due-window assignment. They proved that the nonregular objective minimization (including the earliness, tardiness, duewindow starting time, and due-window size) can be solved in polynomial time. Li and Zhao [29] studied the singlemachine group scheduling problem with multiple duewindows assignment. The objective is to determine an optimal sequence for both groups and jobs, and optimal due-windows such that the total cost of earliness, tardiness, and due-windows assignment is minimized. They showed that the problem can be solved in polynomial time. "The application of problems with positional-dependent weights can be found in many practical settings, such as the busyness of production services often changes with time. The weight of the processing queue can be increased when the production efficiency of a certain period of time needs to be improved. For example, in the Didi taxi dispatching (a similar mode to Uber), orders placed in the morning offer a higher bonus to the driver, which can effectively improve customer satisfaction in these locations by better meeting the needs of customers going to work in the morning [30]." In this article, the results of Wang et al. [21] are extended to the case of the due-window assignment with positiondependent weights (Wang et al. [24], Wang et al. [25], and Wang et al. [31]). In other words, this article studies the group scheduling with the due-window assignment and position-dependent weights, i.e., our model is more general and covers the results of Wang et al. [21], Wang et al. [24], and Wang et al. [25].

The rest of the study is organized as follows: in Section 2, the model and problem is formulated. In Section 3, we present several results of the optimal solution. In Section 4, some examples are given. In Section 5, the conclusions are summarized.

\section{Problem Formulation}

The notations used throughout this article are tabulated in Table 1.
TABLE 1: List of notations.

\begin{tabular}{|c|c|}
\hline Notations & Definitions \\
\hline$N$ & Number of jobs \\
\hline$m$ & Number of groups \\
\hline$T_{l}(l=1,2, \ldots, n)$ & Index of job \\
\hline$\widetilde{G}_{h}(h=1,2, \ldots, m)$ & Index of group \\
\hline$n_{h}$ & Number of group $\widetilde{G}_{h}$ \\
\hline$T_{h, l}$ & Job $T_{l}$ of group $\widetilde{G}_{h}$ \\
\hline$s_{h}$ & Setup time of group $\widetilde{G}_{h}$ \\
\hline & Processing time of job $T_{h, l}$ \\
\hline$\left\langle d_{h, l}^{\prime}, d_{h, l}^{\prime \prime}\right\rangle$ & Due-window of job $T_{h, l}$ \\
\hline$d_{h, l}^{\prime}$ & Due-window starting time of job $T_{h, l}$ \\
\hline & Due-window finishing time of job $T_{h, l}$ \\
\hline$D_{h, l}^{n, l}=d_{h, l}^{\prime \prime}-d_{h, l}^{\prime}$ & Due-window size of job $T_{h, l}$ \\
\hline$C_{h, l}$ & Completion time of job $T_{h, l}$ \\
\hline$d_{h}^{\prime}\left(d_{h}^{\prime \prime}\right)$ & $\begin{array}{l}\text { Starting (finishing) time of common due- } \\
\text { window in group } \widetilde{G}_{h}\end{array}$ \\
\hline$q_{h}^{\prime}\left(q_{h}^{\prime \prime}\right)$ & $\begin{array}{l}\text { Common flow allowance of slack due- } \\
\text { window in group } \widetilde{G}_{h}\end{array}$ \\
\hline$\xi$ & An optimal schedule of jobs \\
\hline$L_{h, l}$ & Lateness penalty of job $T_{h, l}$ \\
\hline$\vartheta_{h, l}^{h, l}\left(l=1,2, \ldots, n_{h}\right)$ & Weight of the $l^{\text {th }}$ position in group $\widetilde{G}_{h}$ \\
\hline$\vartheta_{h, 0}$ & Unit cost of $d_{h, l}^{\prime}$ \\
\hline$\vartheta_{h, n_{h}+1}$ & Unit cost of $D_{h, l}$ \\
\hline CONDW & Common due-window assignment \\
\hline SLKDW & Slack due-window assignment \\
\hline DIFDW & Different due-window assignment \\
\hline$[l]$ & Job scheduled in the $l^{\text {th }}$ position \\
\hline$\Phi_{h, l}$ & Weight cost of $l^{\text {th }}$ position in group $\widetilde{G}_{h}$ \\
\hline$\Omega_{h}^{n, t}$ & Weight factor of group $\widetilde{G}_{h}$ \\
\hline
\end{tabular}

There are $N$ jobs ready to be processed on a single machine, and all the jobs are divided (grouped) into $m$ groups $\left\{\widetilde{G}_{1}, \widetilde{G}_{2}, \ldots, \widetilde{G}_{m}\right\}$ in advance. All the jobs are available at time 0 and job preemption is not allowed. Each group $\widetilde{G}_{h}$ has $n_{h}$ jobs, i.e., $\left\{T_{h, 1}, T_{h, 2}, \ldots, T_{h, n_{h}}\right\}$, where $T_{h, l}$ denotes the job $T_{l}$ of group $\widetilde{G}_{h}$ and $n_{1}+n_{2}+\cdots+n_{m}=N$ $\left(h=1,2, \ldots, m, l=1,2, \ldots, n_{h}\right)$. The jobs in the same group must be processed in succession and do not need setup times, and each group $\widetilde{G}_{h}$ requires an independent setup time $s_{h}$. Each job $T_{h, l}$ has a processing time $p_{h, l}$ and a duewindow $\left\langle d_{h, l}^{\prime}, d_{h, l}^{\prime \prime}\right\rangle$, where $d_{h, l}^{\prime} \leq d_{h, l}^{\prime \prime}, d_{h, l}^{\prime}\left(d_{h, l}^{\prime \prime}\right)$ is the duewindow starting (finishing) time of job $T_{h, l}$ in group $\widetilde{G}_{h}$, $D_{h, l}=d_{h, l}^{\prime \prime}-d_{h, l}^{\prime}$ is due-window size, and both $d_{h, l}^{\prime}$ and $d_{h, l}^{\prime \prime}$ are decision variables. The due-window is assignable according to the following three methods:

(1) The common due-window (CONDW) assignment: all jobs in group $\widetilde{G}_{h}$ are assigned the same duewindow, i.e., $\quad d_{h, l}^{\prime}=d_{h}^{\prime} \quad$ and $\quad d_{h, l}^{\prime \prime}=d_{h}^{\prime \prime}$ $\left(h=1,2, \ldots, m, l=1,2, \ldots, n_{h}\right)$

(2) The slack due-window (SLKDW) assignment: $d_{h, l}^{\prime}=$ $p_{h, l}+q_{h}^{\prime}$ and $d_{h, l}^{\prime \prime}=p_{h, l}+q_{h}^{\prime \prime} \quad(h=1,2, \ldots, m, l=$ $\left.1,2, \ldots, n_{h}\right)$

(3) The different due-window (DIFDW) assignment: the due-window $\left\langle d_{h, l}^{\prime}, d_{h, l}^{\prime \prime}\right\rangle$ for all jobs of group $\widetilde{G}_{h}$ is assigned with no restrictions $(h=1,2, \ldots, m$, $\left.l=1,2, \ldots, n_{h}\right)$ 
Let $C_{h, l}$ be the completion time of job $T_{h, l}$. The objective of the study is to determine $d_{h, l}^{\prime}$ and $d_{h, l}^{\prime \prime}$ (i.e., for CONDW, determine $d_{h}^{\prime}$ and $d_{h}^{\prime \prime}$; for SLKDW, determine $q_{h}^{\prime}$ and $q_{h}^{\prime \prime}$; and for DIFDW, determine $d_{h, l}^{\prime}, d_{h, l}^{\prime \prime}$ of all jobs) and an optimal schedule $\xi$ to minimize a cost function that comprises lateness (earliness-tardiness) penalties, due-window starting time, and due-window size costs, i.e.,

$$
F\left(\xi, d_{h, l}^{\prime}, d_{h, l}^{\prime \prime}\right)=\sum_{h=1}^{m} \sum_{l=1}^{n_{h}}\left(\vartheta_{h, l} L_{\xi(h,[l])}+\vartheta_{h, 0} d_{\xi(h,[l])}^{\prime}+\vartheta_{h, n_{h}+1} D_{\xi(h,[l])}\right),
$$

where $\vartheta_{h, l}$ is the weight of the $l^{\text {th }}$ position in group $\widetilde{G}_{h}$ (i.e., position-dependent weights, Wang et al. [24], Wang et al. [25], and Wang et al. [31]), $\xi(h,[l])$ is the job scheduled in the $l^{\text {th }}$ position in group $\widetilde{G}_{h}, \vartheta_{h, 0}$ denotes the unit cost of $d_{h, l}^{\prime}$, and $\vartheta_{h, n_{h}+1}$ denotes the unit cost of $D_{h, l}$, and the lateness (earliness-tardiness) of job $T_{\xi(h,[l])}$ is

$$
L_{\xi(h,[l])}= \begin{cases}d_{\xi(h,[l])}-C_{\xi(h,[l])}, & \text { for } d_{\xi(h,[l])}>C_{\xi(h,[l])}, \\ 0, & \text { for } d_{\xi(h,[l])} \leq C_{\xi(h,[l])} \leq d_{\xi(h,[l])}, \\ C_{\xi(h,[l])}-d_{\xi(h,[l])}, & \text { for } C_{\xi(h,[l])}>d_{\xi(h,[l])} .\end{cases}
$$

By using the three-field notation (Graham etv al. [32]), the problem can be denoted by

$$
1|\mathrm{GS}, A| \sum_{h=1}^{m} \sum_{l=1}^{n_{h}}\left(\vartheta_{h, l} L_{\xi(h,[l])}+\vartheta_{h, 0} d_{\xi(h,[l])^{\prime}}+\vartheta_{h, n_{h}+1} D_{\xi(h,[l])}\right),
$$

where $A \in\{C O N D W$, SLKDW, DIFDW $\}$.

Procurement sharing of sustainable and regular products: self-competition and sharing incentives.

\section{Preliminary Results}

It is clear that there exists an optimal schedule that starts at time 0 and contains 0 machine idle times.

\subsection{CONDW Method}

Lemma 1. For a given schedule $\xi$, the optimal values $d_{h}^{\prime}$ and $d_{h}^{\prime \prime}$ coincide with the job completion times of group $\widetilde{G}_{h}(h=1,2, \ldots, m)$.

Proof. For the given schedule $\xi$, under group $\widetilde{G}_{h}$, we assume that

$$
\begin{aligned}
& d_{h}^{\prime}=C_{\xi\left(h,\left[g_{h}\right]\right)}=S_{h}+s_{h}+\sum_{l=1}^{g_{h}} p_{h,[l]}, \\
& d_{h}^{\prime \prime}=C_{\xi\left(h,\left[k_{h}\right]\right)}=S_{h}+s_{h}+\sum_{l=1}^{k_{h}} p_{h,[l]},
\end{aligned}
$$

where $S_{h}$ denotes the starting time of group $\widetilde{G}_{h}, g_{h}$ and $k_{h}$ mean the $g_{h}{ }^{\text {th }}$ and $k_{h}$ th positions of group $\widetilde{G}_{h}$, respectively $\left(g_{h} \leq k_{h}\right)$. Consider that there exists an optimal schedule without the stated property, i.e., $d_{h}^{\prime}=S_{h}+s_{h}+\sum_{\theta=1}^{g_{h}} p_{h,[\theta]}+$ $x$ and $d_{h}^{\prime \prime}=S_{h}+s_{h}+\sum_{\theta=1}^{k_{h}} p_{h,[\theta]}+y$, where $0 \leq x \leq p_{h,\left[g_{h}+1\right]}$ and $0 \leq y \leq p_{h,\left[k_{k}+1\right]}$.

For group $G_{h}$, the total cost is

$$
\begin{aligned}
F_{h}(x, y)= & \sum_{l=1}^{n_{h}}\left(\vartheta_{h, l} L_{\xi(h,[l])}+\vartheta_{h, 0} d_{\xi(h,[l])^{\prime}}+\vartheta_{h, n_{h}+1} D_{\xi(h,[l])}\right) \\
= & \sum_{l=1}^{g_{h}} \vartheta_{h, l}\left(d_{h^{\prime}}-C_{\xi(h,[l])}\right)+\sum_{l=k_{h}+1}^{n_{h}} \vartheta_{h, l}\left(C_{\xi(h,[l])}-d_{h^{\prime \prime}}\right)+n_{h} \vartheta_{h, 0} d_{h^{\prime}}+n_{h} \vartheta_{h, n_{h}+1}\left(d_{h^{\prime \prime}}-d_{h^{\prime}}\right) \\
= & \sum_{l=1}^{g_{h}} \vartheta_{h, l}\left(\sum_{\theta=1}^{g_{h}} p_{h,[\theta]}+x-\sum_{\theta=1}^{l} p_{h,[\theta]}\right) \\
& +\sum_{l=k_{h}+1}^{n_{h}} \vartheta_{h, l}\left(\sum_{\theta=1}^{l} p_{h,[\theta]}-\sum_{\theta=1}^{k_{h}} p_{h,[\theta]}-y\right)+n_{h} \vartheta_{h, 0}\left(s_{h}+s_{h}+\sum_{\theta=1}^{g_{h}} p_{h,[\theta]}+x\right)+n_{h} \vartheta_{h, n_{h}+1}\left(\sum_{\theta=1}^{k_{h}} p_{h,[\theta]}+y-\sum_{\theta=1}^{g_{h}} p_{h,[\theta]}-x\right) \\
= & \sum_{l=1}^{g_{h}} \vartheta_{h, l}\left(\sum_{\theta=l+1}^{g_{h}} p_{h,[\theta]}+x\right) \\
& +\sum_{l=k_{h}+1}^{n_{h}} \vartheta_{h, l}\left(\sum_{\theta=k_{h}+1}^{l} p_{h,[\theta]}-y\right)+n_{h} \vartheta_{h, 0}\left(s_{h}+s_{h}+\sum_{\theta=1}^{g_{h}} p_{h,[\theta]}+x\right)+n_{h} \vartheta_{h, n_{h}+1}\left(\sum_{\theta=g_{h}+1}^{k_{h}} p_{h,[\theta]}+y-x\right) \\
= & \sum_{l=1}^{g_{h}} \vartheta_{h, l}\left(\sum_{\theta=l+1}^{g_{h}} p_{h,[\theta]}\right)+\sum_{l=k_{h}+1}^{n_{h}} \vartheta_{h, l}\left(\sum_{\theta=k_{h}+1}^{l} p_{h,[\theta]}\right)+n_{h} \vartheta_{h, 0}\left(s_{h}+s_{h}+\sum_{\theta=1}^{g_{h}} p_{h,[\theta]}\right)+n_{h} \vartheta_{h, n_{h}+1}\left(\sum_{\theta=g_{h}+1}^{k_{h}} p_{h,[\theta]}\right) \\
& +\left(\sum_{l=1}^{g_{h}} \vartheta_{h, l}+n_{h} \vartheta_{h, 0}-n_{h} \vartheta_{h, n_{h}+1}\right) x+\left(n_{h} \vartheta_{h, n_{h}+1}-\sum_{l=k_{h}+1}^{n_{h}} \vartheta_{h, l}\right) y .
\end{aligned}
$$


From (5), we see that the term $F_{h}(x, y)$ is a linear function of $x$ and $y$; hence, we can either decrease $x$ and $y$ to 0 or increase them to $p_{h,\left[g_{h}+1\right]}$ and $p_{h,\left[k_{h}+1\right]}$, respectively, to obtain a lower cost. This completes the proof.

Lemma 2. For a given schedule $\xi$, the optimal values $d_{h}^{\prime}=$ $S_{h}+s_{h}+\sum_{l=1}^{g_{h}} p_{h,[l]}$ (where $\sum_{l=0}^{g_{h}-1} \vartheta_{h, l} \leq \vartheta_{h, n_{h}+1} \leq \sum_{l=0}^{g_{h}} \vartheta_{h, l}$ ) and $d_{h}^{\prime \prime}=S_{h}+s_{h}+\sum_{l=1}^{k_{h}} p_{h,[l]} \quad$ (where $\quad \sum_{l=k_{h}+1}^{n_{h}} \vartheta_{h, l} \leq \vartheta_{h, n_{h}+1} \leq$ $\left.\sum_{l=k_{h}}^{n_{h}} \vartheta_{h, l}\right)$.

Proof. By the technique of small perturbations, the result can be easily obtained.

For a given schedule $\xi$, the total cost of all the jobs within $\widetilde{G}_{h}(h=1, \ldots, m)$ is

$$
\begin{aligned}
F_{h}\left(\xi, d_{h}^{\prime}, d_{h}^{\prime \prime}\right)= & \sum_{l=1}^{g_{h}} \vartheta_{h, l}\left(\sum_{\theta=l+1}^{g_{h}} p_{h,[\theta]}\right) \\
& +\sum_{l=k_{h}+1}^{n_{h}} \vartheta_{h, l}\left(\sum_{\theta=k_{h}+1}^{l} p_{h,[\theta]}\right) \\
& +n_{h} \vartheta_{h, 0}\left(S_{h}+s_{h}+\sum_{\theta=1}^{g_{h}} p_{h,[\theta]}\right) \\
& +n_{h} \vartheta_{h, n_{h}+1}\left(\sum_{\theta=g_{h}+1}^{k_{h}} p_{h,[\theta]}\right) \\
= & \sum_{l=1}^{n_{h}} \Phi_{h, l} p_{h,[l]}+n_{h} \vartheta_{h, 0}\left(S_{h}+s_{h}\right),
\end{aligned}
$$

where

$$
\Phi_{h, l}= \begin{cases}n_{h} \vartheta_{h, 0}+\sum_{\theta=1}^{l-1} \vartheta_{h, \theta}, & l=1,2, \ldots, g_{h}, \\ n_{h} \vartheta_{h, n_{h}+1}, & l=g_{h}+1, g_{h}+2, \ldots, k_{h}, \\ \sum_{\theta=l}^{n_{h}} \vartheta_{h, \theta}, & l=k_{h}+1, k_{h}+2, \ldots, n_{h} .\end{cases}
$$

From (6) and HLP rule (Hardy et al. [33], i.e., the sum of products $\sum_{j=1}^{n} x_{j} y_{j}$ is minimized if the sequence $x_{1}, x_{2}, \ldots, x_{n}$ is ordered nondecreasingly and the sequence $y_{1}, y_{2}, \ldots, y_{n}$ is ordered nonincreasingly, or vice versa, and it is maximized if the sequences are ordered in the same way), minimizing $\sum_{l=1}^{n_{h}} \Phi_{h, l} p_{h,[l]}$ can be obtained by arranging the elements of the $\Phi_{h, l}$ and $p_{h, l}$ vectors in opposite orders. The term $n_{h} \vartheta_{h, 0}\left(S_{h}+s_{h}\right)$ is only dependent on the starting time of the group $\widetilde{G}_{h}(h=1, \ldots, m)$.

Lemma 3. For the problem $1|G S, C O N D W| \sum_{h=1}^{m} \sum_{l=1}^{n_{h}}$ $\left(\vartheta_{h, l} L_{\xi(h,[l])}+\vartheta_{h, 0} d_{\xi(h,[l])}^{\prime}+\vartheta_{h, n_{h}+1} D_{\xi(h,[l])}\right)$, the optimal group sequence can be obtained by arranging the groups in a nondecreasing order of $\Omega_{h}\left(s_{h}+\sum_{l=1}^{n_{h}} p_{h,[l]}\right) /\left(n_{h} \vartheta_{h, 0}\right)$, $h=1,2, \ldots, m$.

Proof. By contradiction, let $\xi=\left[\pi, \widetilde{G}_{f}, \widetilde{G}_{h}, \rho\right]$ be an optimal schedule, such that

$$
\frac{s_{f}+\sum_{l=1}^{n_{f}} p_{f,[l]}}{n_{f} \vartheta_{f, 0}}>\frac{s_{h}+\sum_{l h=1}^{n_{h}} p_{h,[l]}}{n_{h} \vartheta_{h, 0}},
$$

where $\pi$ and $\rho$ are the partial sequences. We now perform an adjacent pairwise interchange of $\widetilde{G}_{f}$ and $\widetilde{G}_{h}$, leaving all other groups in their original positions, to derive a new schedule $\xi^{\prime}=\left[\pi, G_{h}, G_{f}, \rho\right]$. Let $B$ denote the completion time of the last job in $\pi$, and it follows that

$$
\begin{aligned}
S_{h}(\xi)= & B+s_{f}+\sum_{l=1}^{n_{f}} p_{f,[l]}, \\
S_{f}\left(\xi^{\prime}\right)= & B+s_{h}+\sum_{l=1}^{n_{h}} p_{h,[l]}, \\
& F(\xi)-F\left(\xi^{\prime}\right) \\
= & n_{f} \vartheta_{f, 0}\left(B+s_{f}\right)+n_{h} \vartheta_{h, 0}\left(B+s_{f}+\sum_{l=1}^{n_{f}} p_{f,[l]}+s_{h}\right) \\
& -n_{h} \vartheta_{h, 0}\left(B+s_{h}\right)-n_{f} \vartheta_{f, 0}\left(B+s_{h}+\sum_{l=1}^{n_{h}} p_{h,[l]}+s_{f}\right) \\
= & n_{h} \vartheta_{h, 0}\left(s_{f}+\sum_{l=1}^{n_{f}} p_{f,[l]}\right)-n_{f} \vartheta_{f, 0}\left(s_{h}+\sum_{l=1}^{n_{h}} p_{h,[l]}\right) \\
> & 0 .
\end{aligned}
$$

This contradicts the optimality of $\xi$ and proves that groups are arranged in a nondecreasing order of $\Omega_{h}=\left(s_{h}+\sum_{l=1}^{n_{h}} p_{h,[l]}\right) /\left(n_{h} \vartheta_{h, 0}\right)$.

Based on the above analysis, the following algorithm can be proposed to solve the problem $1|\mathrm{GS}, \mathrm{CONDW}| \sum_{h=1}^{m} \sum_{l=1}^{n_{h}}$ $\left(\vartheta_{h, l} L_{\xi(h,[l])}+\vartheta_{h, 0} d_{\xi(h,[l])}^{\prime}+\vartheta_{h, n_{h}+1} D_{\xi(h,[l])}\right)$.

Theorem 1. The problem $1|G S, C O N D W| \sum_{h=1}^{m} \sum_{l=1}^{n_{h}}$ $\left(\vartheta_{h, l} L_{\xi(h,[l])}+\vartheta_{h, 0} d_{\xi(h,[l])}+\vartheta_{h, n_{h}+1} D_{\xi(h,[l])}\right)$ can be solved by Algorithm 1 in $O(N \log N)$ time.

Proof. The correctness of Algorithm 1 follows Lemmas 1-3 and the above analysis. Steps 1 and 4 need $O(m)$ time. Step 2 needs $O\left(\sum_{h=1}^{m}\left(n_{h} \log n_{h}\right)\right) \leq O(N \log N)$ time. Step 3 needs $O(m \log m) \leq O(N \log N)$ time $(m<N)$. Thus, the total time of Algorithm 1 is $O(N \log N)$.

3.2. SLKDW Method. Similar to Subsection 3.1, we have the following.

Lemma 4. For a given schedule $\xi$, the optimal values $q_{h}^{\prime}$ and $q_{h}^{\prime \prime}$ coincide with the job completion times of group $G_{h}(h=1,2, \ldots, m)$.

Lemma 5. For a given schedule $\xi$, the optimal values $q_{h}^{\prime}=$ $S_{h}+s_{h}+\sum_{l=1}^{g_{h}-1} p_{h,[l]} \quad$ (where $\sum_{l=0}^{g_{h}-1} \vartheta_{h, l} \leq \vartheta_{h, n_{h}+1} \leq \sum_{l=0}^{g_{h}} \vartheta_{h, l}$ ) and $q_{h}^{\prime \prime}=S_{h}+s_{h}+\sum_{l=1}^{k_{h}-1} p_{h,[l]} \quad$ (where $\sum_{l=k_{h}+1}^{n_{h, l}} \vartheta_{l} \leq$ $\left.\vartheta_{h, n_{h}+1} \leq \sum_{l=k_{l}}^{n_{h}} \vartheta_{h, l}\right)$. 
Step 1: calculate $g_{h}$ and $k_{h}$ by Lemma $2, h=1,2, \ldots, m$

Step 2: arrange the jobs of each group by the HLP rule (vectors $\Phi_{h, l}(7)$ and $p_{h, l}$ )

Step 3: by Lemma 3, arrange the groups in a nondecreasing order of $\Omega_{h}=\left(s_{h}+\sum_{l=1}^{n_{h}} p_{h,[l]}\right) /\left(n_{h} \vartheta_{h, 0}\right)(h=1,2, \ldots, m)$

Step 4: according to Lemma 2, calculate $d_{h}^{\prime}$ and $d_{h}^{\prime \prime}(h=1,2, \ldots, m)$

Algorithm 1

For a given schedule $\xi$, the total cost of all the jobs within

$\widetilde{G}_{h}(h=1, \ldots, m)$ is

$$
\begin{aligned}
& F_{h}\left(\xi, q_{h^{\prime}}, q_{h^{\prime \prime}}\right)=\sum_{l=1}^{n_{h}}\left(\vartheta_{h, l} L_{\xi(h,[l])}+\vartheta_{h, 0} d_{\xi(h,[l])^{\prime}}+\vartheta_{h, n_{h}+1} D_{\xi(h,[l])}\right) \\
& =\sum_{l=1}^{g_{h}} \vartheta_{h, l}\left(d_{\xi(h,[l])^{\prime}}-C_{\xi(h,[l])}\right)+\sum_{l=k_{h}+1}^{n_{h}} \vartheta_{h, l}\left(C_{\xi(h,[l])}-d_{\xi(h,[l])^{\prime \prime}}\right) \\
& +\vartheta_{h, 0} \sum_{l=1}^{n_{h}} d_{\xi(h,[l])^{\prime}}+\vartheta_{h, n_{h}+1} \sum_{l=1}^{n_{h}}\left(d_{\xi(h,[l])^{\prime \prime}}-d_{\xi(h,[l])^{\prime}}\right) \\
& =\sum_{l=1}^{g_{h}} \vartheta_{h, l}\left(p_{h,[l]}+q_{h^{\prime}}-C_{\xi(h,[l])}\right)+\sum_{l=k_{h}+1}^{n_{h}} \vartheta_{h, l}\left(C_{\xi(h,[l])}-p_{(h,[l])}-q_{h^{\prime \prime}}\right) \\
& +\vartheta_{h, 0} \sum_{l=1}^{n_{h}}\left(p_{h,[l]}+q_{h^{\prime}}\right)+n_{h} \vartheta_{h, n_{h}+1}\left(q_{h^{\prime \prime}}-q_{h^{\prime}}\right) \\
& =\sum_{l=1}^{g_{h}} \vartheta_{h, l}\left(\sum_{\theta=1}^{g_{h}-1} p_{h,[\theta]}-\sum_{\theta=1}^{l-1} p_{h,[\theta]}\right)+\sum_{l=k_{h}+1}^{n_{h}} \vartheta_{h, l}\left(\sum_{\theta=1}^{l-1} p_{h,[\theta]}-\sum_{\theta=1}^{k_{h}-1} p_{h,[\theta]}\right) \\
& +\vartheta_{h, 0} \sum_{l=1}^{n_{h}} p_{h,[l]}+n_{h} \vartheta_{h, 0}\left(S_{h}+s_{h}+\sum_{\theta=1}^{g_{h}-1} p_{h,[\theta]}\right)+n_{h} \vartheta_{h, n_{h}+1}\left(\sum_{\theta=1}^{k_{h}-1} p_{h,[\theta]}-\sum_{\theta=1}^{g_{h}-1} p_{h,[\theta]}\right) \\
& =\sum_{l=1}^{g_{h}} \vartheta_{h, l}\left(\sum_{\theta=l}^{g_{h}-1} p_{h,[\theta]}\right)+\sum_{l=k_{h}+1}^{n_{h}} \vartheta_{h, l}\left(\sum_{\theta=k_{h}}^{l-1} p_{h,[\theta]}\right) \\
& +\vartheta_{h, 0} \sum_{l=1}^{n_{h}} p_{h,[l]}+n_{h} \vartheta_{h, 0}\left(S_{h}+s_{h}+\sum_{\theta=1}^{g_{h}-1} p_{h,[\theta]}\right)+n_{h} \vartheta_{h, n_{h}+1}\left(\sum_{\theta=g_{h}}^{k_{h}-1} p_{h,[\theta]}\right) \\
& =\sum_{l=1}^{n_{h}} \Phi_{h, l} p_{h,[l]}+n_{h} \vartheta_{h, 0}\left(S_{h}+s_{h}\right)
\end{aligned}
$$

where

$$
\Phi_{h, l}= \begin{cases}\left(n_{h}+1\right) \vartheta_{h, 0}+\sum_{\theta=1}^{l} \vartheta_{h, \theta}, & l=1,2, \ldots, g_{h}-1, \\ \vartheta_{h, 0}+n_{h} \vartheta_{h, n_{h}+1}, & l=g_{h}, g_{h}+1, \ldots, k_{h}-1, \\ \vartheta_{h, 0}+\sum_{\theta=l+1}^{n_{h}} \vartheta_{h, \theta}, & l=k_{h}, k_{h}+1, \ldots, n_{h}-1 \\ \vartheta_{h, 0}, & l=n_{h} .\end{cases}
$$

From (10) and Subsection 3.1, the optimal schedule of each group can be obtained by the HLP rule.
Lemma 6. For the problem 1|GS,SLKDW $\mid \sum_{h=1}^{m} \sum_{l=1}^{n_{h}}$ $\left(\vartheta_{h, l} L_{\xi(h,[l])}+\vartheta_{h, 0} d_{\xi(h,[l])}+\vartheta_{h, n_{h}+1} D_{\xi(h,[l])}\right)$, the optimal group sequence can be obtained by arranging the groups in a nondecreasing order of $\Omega_{h}=\left(s_{h}+\sum_{l=1}^{n_{h}} p_{h,[l]}\right) /$ $\left(n_{h} \vartheta_{h, 0}\right), h=1,2, \ldots, m$.

Based on the above analysis, the following algorithm can be proposed to solve the problem $1|G S, S L K D W| \sum_{h=1}^{m} \sum_{l=1}^{n_{h}}$ $\left(\vartheta_{h, l} L_{\xi(h,[l])}+\vartheta_{h, 0} d_{\xi(h,[l])}+\vartheta_{h, n_{h}+1} D_{\xi(h,[l])}\right)$.

Theorem 2. The problem 1|GS, SLKDW $\mid \sum_{h=1}^{m} \sum_{l=1}^{n_{h}}$ $\left(\vartheta_{h, l} L_{\xi(h,[l])}+\vartheta_{h, 0} d_{\xi(h,[l])}+\vartheta_{h, n_{h}+1} D_{\xi(h,[l])}\right)$ can be solved by Algorithm 2 in $O(N \log N)$ time. 
Step 1: calculate $g_{h}$ and $k_{h}$ by Lemma $5, h=1,2, \ldots, m$

Step 2: arrange the jobs of each group by the HLP rule (vectors $\Phi_{h, l}(11)$ and $p_{h, l}$ )

Step 3: by Lemma 6, arrange the groups in a nondecreasing order of $\Omega_{h}=\left(s_{h}+\sum_{l=1}^{n_{h}} p_{h,[l]}\right) /\left(n_{h} \vartheta_{h, 0}\right),(h=1,2, \ldots, m)$

Step 4: according to Lemma 5 , calculate $q_{h}^{\prime}$ and $q_{h}^{\prime \prime}(h=1,2, \ldots, m)$

\section{Algorithm 2}

\subsection{DIFDW Method}

Lemma 7. For the DIFDW method, there exists an optimal sequence, such that $d_{h, l}^{\prime} \leq d_{h, l}^{\prime} \leq C_{h, l}$.

Proof. Similar to the proof of Wang et al. [24].

Lemma 8. For the DIFDW method, let $\xi$ be a given sequence, and the optimal $d_{\xi(h,[j])}$ and $d_{\xi(h,[j])}$ " of job $J_{\xi(h,[j])}$ can be given as follows:

(1) If $\min _{d_{\xi(h,[j])}}\left\{\vartheta_{h, 0}, \vartheta_{h, l}, \vartheta_{h, h_{h}+1}\right\}=\vartheta_{h, 0}$, then $d_{\xi(h,[j])}{ }^{\prime}=$
$d_{(j,)}$

(2) If $\min \left\{\vartheta_{h, 0}, \vartheta_{h, l}, \vartheta_{h, n_{h}+1}\right\}=\vartheta_{h, l}$, then $d_{\xi(h,[j])}=$ $d_{\xi(h,[j])}^{\prime \prime}=0$

(3) If $\min \left\{\vartheta_{h, 0}, \vartheta_{h, l}, \vartheta_{h, n_{h}+1}\right\}=\vartheta_{h, n_{h}+1}$, then $d_{\xi(h,[j])}=$ $0, d_{\xi(h,[j])}=C_{\xi(h,[j])}$

Proof. Similar to the proof of Wang et al. [24].

From Lemma 8, the cost for job $J_{\xi(h,[l])}$ is

$$
F_{\xi(h,[l])}=\psi_{h, l} C_{\xi(h,[l])},
$$

where $\psi_{h, l}=\min \left\{\vartheta_{h, 0}, \vartheta_{h, l}, \vartheta_{h, n_{h}+1}\right\}$. The total cost of all the jobs within $\widetilde{G}_{h}$ is

$$
\begin{aligned}
F_{h}\left(\xi, d_{\xi(h,[j])}, d_{\xi(h,[j])}^{\prime \prime}\right) & =\sum_{l a=1}^{n_{h}} \psi_{h, l} C_{\xi(h,[l])} \\
& =\sum_{l=1}^{n_{h}} \Phi_{h, l} p_{h,[l]}+\left(S_{h}+s_{h}\right) \sum_{l=1}^{n_{h}} \psi_{h, l},
\end{aligned}
$$

where

$$
\Phi_{h, l}=\left(n_{h}-l+1\right) \psi_{h, l} .
$$

From (13) and Subsection 3.1, the optimal schedule of each group can be obtained by the HLP rule.

Lemma 9. For the problem 1|GS, DIFDW $\mid \sum_{h=1}^{m} \sum_{l=}$ $1^{n_{h}}\left(\vartheta_{h, l} L_{\xi(h,[l])}+\vartheta_{h, 0} d_{\xi(h,[l])}+\vartheta_{h, n_{h}+1} D_{\xi(h,[l])}\right)$, the optimal group sequence can be obtained by arranging the groups in a nondecreasing order of $\Omega_{h}=\left(s_{h}+\sum_{l=1}^{n_{h}} p_{h,[l]}\right) /\left(\sum_{l=1}^{n_{h}} \psi_{h, l}\right)$, $h=1,2, \ldots, m$.

Based on the above analysis, the following algorithm can be proposed to solve the problem $1|G S, S L K D W| \sum_{h=1}^{m} \sum_{l=1}^{n_{h}}$ $\left(\vartheta_{h, l} L_{\xi(h,[l])}+\vartheta_{h, 0} d_{\xi(h,[l])}+\vartheta_{h, n_{h}+1} D_{\xi(h,[l])}\right)$.
Theorem 3. The problem $1|G S, D I F D W| \sum_{h=1}^{m} \sum_{l=1}^{n_{h}}$ $\left(\vartheta_{h, l} L_{\xi(h,[l])}+\vartheta_{h, 0} d_{\xi(h,[l])}+\vartheta_{h, n_{h}+1} D_{\xi(h,[l])}\right)$ can be solved by Algorithm 3 in $O(N \log N)$ time.

\section{Number Examples}

Example 1. For the problem 1|GS, CONDW| $\sum_{h=1}^{m} \sum_{l=1}^{n_{h}}$ $\left(\vartheta_{h, l} L_{\xi(h,[l])}+\vartheta_{h, 0} d_{\xi(h,[l])}+\vartheta_{h, n_{h}+1} D_{\xi(h,[l])}\right)$, consider $n=$ $13, m=3, n_{1}=4, n_{2}=4, n_{3}=5, \widetilde{G}_{1}: s_{1}=5, p_{1,1}=4, p_{1,2}=3$, $p_{1,3}=6, p_{1,4}=2, \vartheta_{1,0}=2, \vartheta_{1,1}=3, \vartheta_{1,2}=5, \vartheta_{1,3}=4, \vartheta_{1,4}=6$, $\vartheta_{1,5}=7 ; \quad \widetilde{G}_{2}: s_{2}=3, \quad p_{2,1}=8, \quad p_{2,2}=2, \quad p_{2,3}=7, \quad p_{2,4}=5$, $\vartheta_{2,0}=6, \vartheta_{2,1}=7, \vartheta_{2,2}=4, \vartheta_{2,3}=5, \vartheta_{2,4}=2, \vartheta_{2,5}=8 ; \widetilde{G}_{3}$ : $s_{3}=6, \quad p_{3,1}=14, \quad p_{3,2}=7, \quad p_{3,3}=5, \quad p_{3,4}=10, \quad p_{3,5}=9$, $\vartheta_{3,0}=3, \vartheta_{3,1}=6, \vartheta_{3,2}=7, \vartheta_{3,3}=5, \vartheta_{3,4}=8, \vartheta_{3,5}=2$, and $\vartheta_{3,6}=12$.

From Algorithm 1, we have the following.

Step 1: $g_{1}=2, k_{1}=3, g_{2}=1, k_{2}=2, g_{3}=2$, and $k_{3}=3$ Step 2: $\Phi_{1,1}=8, \quad \Phi_{1,2}=11, \quad \Phi_{1,3}=28, \quad \Phi_{1,4}=6$; $\Phi_{2,1}=24, \quad \Phi_{2,2}=32, \quad \Phi_{2,3}=7, \quad \Phi_{2,4}=2 ; \quad \Phi_{3,1}=15$, $\Phi_{3,2}=21, \Phi_{3,3}=60, \Phi_{3,4}=10$, and $\Phi_{3,5}=2$. The optimal sequence of jobs within each group is $\widetilde{G}_{1}$ : $T_{1, l} \longrightarrow T_{1,2} \longrightarrow T_{1,4} \longrightarrow T_{1,3} ; \widetilde{G}_{2}: T_{2,4} \longrightarrow T_{2,2} \longrightarrow$ $T_{2,3} \longrightarrow T_{2,1} ;$ and $\widetilde{G}_{3}: T_{3,5} \longrightarrow T_{3,2} \longrightarrow T_{3,3} \longrightarrow$ $T_{3,4} \longrightarrow T_{3,1}$.

Step 3: $\Omega_{1}=5 / 2, \Omega_{2}=25 / 24$, and $\Omega_{3}=51 / 15$; the optimal group sequence is $\widetilde{G}_{2} \longrightarrow \widetilde{G}_{1} \longrightarrow \widetilde{G}_{3}$.

Step 4: $d_{2}^{\prime}=8, d_{2}^{\prime \prime}=10 ; d_{1}^{\prime}=37, d_{1}^{\prime \prime}=39 ; d_{3}^{\prime}=67$, and $d_{3}^{\prime \prime}=72$

Example 2. For the problem $1|\mathrm{GS}, \mathrm{SLKDW}| \sum_{h=1}^{m} \sum_{l=}$ $1^{n_{h}}\left(\vartheta_{h, l} L_{\xi(h,[l])}+\vartheta_{h, 0} d_{\xi(h,[l])}+\vartheta_{h, n_{h}+1} D_{\xi(h,[l])}\right)$, the data are the same as in Example 1.

From Algorithm 2, we have the following.

Step 1: $g_{1}=2, k_{1}=3, g_{2}=1, k_{2}=2, g_{3}=2$, and $k_{3}=3$ Step 2: $\Phi_{1,1}=13, \quad \Phi_{1,2}=30, \quad \Phi_{1,3}=8, \quad \Phi_{1,4}=2$; $\Phi_{2,1}=38, \quad \Phi_{2,2}=13, \quad \Phi_{2,3}=8, \quad \Phi_{2,4}=6 ; \quad \Phi_{3,1}=24$, $\Phi_{3,2}=63, \Phi_{3,3}=13, \Phi_{3,4}=5$, and $\Phi_{3,5}=3$. The optimal sequence of jobs within each group is $\widetilde{G}_{1}$ : $T_{1,2} \longrightarrow T_{1,4} \longrightarrow T_{1,1} \longrightarrow T_{1,3} ; \widetilde{G}_{2}: T_{2,2} \longrightarrow T_{2,4} \longrightarrow$ $T_{2,3} \longrightarrow T_{2,1}$; and $\widetilde{G}_{3}: T_{3,2} \longrightarrow T_{3,3} \longrightarrow T_{3,5} \longrightarrow$ $T_{3,4} \longrightarrow T_{3,1}$.

Step 3: $\Omega_{1}=5 / 2, \Omega_{2}=25 / 24$, and $\Omega_{3}=51 / 15$; the optimal group sequence is $\widetilde{G}_{2} \longrightarrow \widetilde{G}_{1} \longrightarrow \widetilde{G}_{3}$.

Step 4: $q_{2}^{\prime}=3, q_{2}^{\prime \prime}=5 ; q_{1}^{\prime}=33, q_{1}^{\prime \prime}=35 ; q_{3}^{\prime}=58$, and $q_{3}^{\prime \prime}=63$ 
Step 1: arrange the jobs of each group by the HLP rule (vectors $\Phi_{h, l}(14)$ and $p_{h, l}$ )

Step 2: arrange the groups in a nondecreasing order of $\Omega_{h}=\left(s_{h}+\sum_{l=1}^{n_{h}} p_{h,[l]}\right) /\left(\sum_{l=1}^{n_{h}} \psi_{h, l}\right),(h=1,2, \ldots, m)$

Step 3: according to Lemma 8 , calculate $d_{\xi(h,[j])}, d_{\xi(h,[j])}, h=1,2, \ldots, m$, and $j=1,2, \ldots, n_{h}$

Algorithm 3

Example 3. For the problem $1|\mathrm{GS}, \mathrm{DIFDW}| \sum_{h=}$ $1^{m} \sum_{l=1}^{n_{h}}\left(\vartheta_{h, l} L_{\xi(h,[l])}+\vartheta_{h, 0} d_{\xi(h,[l])}+\vartheta_{h, n_{h}+1} D_{\xi(h,[l])}\right)$, the data are the same as in Example 1.

From Algorithm 3, we have the following.

Step 1: $\Phi_{1,1}=8, \Phi_{1,2}=6, \Phi_{1,3}=4, \Phi_{1,4}=2 ; \Phi_{2,1}=24$, $\Phi_{2,2}=12, \Phi_{2,3}=10, \Phi_{2,4}=2 ; \quad \Phi_{3,1}=15, \Phi_{3,2}=12$, $\Phi_{3,3}=9, \Phi_{3,4}=6$, and $\Phi_{3,5}=2$. The optimal sequence of jobs within each group is $\widetilde{G}_{1}: T_{1,4} \longrightarrow$ $T_{1,2} \longrightarrow T_{1,1} \longrightarrow T_{1,3} ; \quad \widetilde{G}_{2}: \quad T_{2,2} \longrightarrow T_{2,4} \longrightarrow$ $T_{2,3} \longrightarrow T_{2,1} ; \quad$ and $\quad \widetilde{G}_{3}: \quad T_{3,3} \longrightarrow T_{3,2} \longrightarrow$ $T_{3,5} \longrightarrow T_{3,4} \longrightarrow T_{3,1}$.

Step 2: $\Omega_{1}=5 / 2, \Omega_{2}=25 / 17$, and $\Omega_{3}=51 / 14$; the optimal group sequence is $\widetilde{G}_{2} \longrightarrow \widetilde{G}_{1} \longrightarrow \widetilde{G}_{3}$.

Step 3: $d_{2,2}{ }^{\prime}=d_{2,2}{ }^{\prime \prime}=7, d_{2,4}{ }^{\prime}=d_{2,4}{ }^{\prime \prime}=0, d_{2,3}{ }^{\prime}=d_{2,3}{ }^{\prime \prime}=0$, $d_{2,1}^{\prime}=d_{2,1}^{\prime \prime}=0 ; \quad d_{1,4}^{\prime}=d_{1,4}^{\prime \prime}=32, \quad d_{1,2}^{\prime}=d_{1,2}^{\prime \prime}=35$, $d_{1,1}^{\prime}=d_{1,1}^{\prime \prime}=39, \quad d_{1,3}^{\prime}=d_{1,3}^{\prime \prime}=45 ; \quad d_{3,3}^{\prime}=d_{3,3}^{\prime \prime}=56$, $d_{3,2}{ }^{\prime}=d_{3,2}{ }^{\prime \prime}=63, d_{3,5}{ }^{\prime}=d_{3,5}{ }^{\prime \prime}=72, d_{3,4}{ }^{\prime}=d_{3,4}{ }^{\prime \prime}=82$, and $d_{3,1}{ }^{\prime}=d_{3,1}{ }^{\prime \prime}=0$

\section{Conclusions}

We extended the classical single-machine scheduling with the due-window assignment and position-dependent weights to the group technology assumption. For the CONDW, SLKDW, and DIFDW assignment methods, our objective is to minimize a cost function including lateness (earliness-tardiness), starting times, and sizes of due-window. Some properties of the above three assignment methods were given, and three algorithms can be proposed in the $O(N \log N)$ time algorithm. A future extension is to the group scheduling in the flowshop, parallel machines setting, or two-stage assembly flowshop. Other future research may study extending the group scheduling to scenario-dependent processing times (Wu et al. [34-36]) or variable processing times (Wang et al. $[37,38]$ ).

\section{Data Availability}

No data were used to support this study.

\section{Conflicts of Interest}

The authors declare that they have no conflicts of interest.

\section{Acknowledgments}

This research was supported by the National Natural Science Regional Foundation of China (71861031 and 72061029).

\section{References}

[1] J. S. Neufeld, J. N. D. Gupta, and U. Buscher, "A comprehensive review of flowshop group scheduling literature," Computers \& Operations Research, vol. 70, pp. 56-74, 2016.

[2] Y.-Y. Lu, J.-J. Wang, and J.-B. Wang, "Single machine group scheduling with decreasing time-dependent processing times subject to release dates," Applied Mathematics and Computation, vol. 234, pp. 286-292, 2014.

[3] N. Yin, L. Kang, and X.-Y. Wang, "Single-machine group scheduling with processing times dependent on position, starting time and allotted resource," Applied Mathematical Modelling, vol. 38, no. 19-20, pp. 4602-4613, 2014.

[4] J.-J. Wang and Y.-J. Liu, "Single-machine bicriterion group scheduling with deteriorating setup times and job processing times," Applied Mathematics and Computation, vol. 242, pp. 309-314, 2014.

[5] J.-B. Wang and J.-J. Wang, "Single machine group scheduling with time dependent processing times and ready times," Information Sciences, vol. 275, pp. 226-231, 2014.

[6] Y. He and L. Sun, "One-machine scheduling problems with deteriorating jobs and position-dependent learning effects under group technology considerations," International Journal of Systems Science, vol. 46, no. 7, pp. 1319-1326, 2015.

[7] Y.-Y. Lu, J.-B. Wang, P. Ji, and H. He, "A note on resource allocation scheduling with group technology and learning effects on a single machine," Engineering Optimization, vol. 49, no. 9, pp. 1621-1632, 2017.

[8] X. Zhang, L. Liao, W. Zhang, T. C. E. Cheng, Y. Tan, and M. Ji, "Single-machine group scheduling with new models of position-dependent processing times," Computers \& Industrial Engineering, vol. 117, pp. 1-5, 2018.

[9] F. Liu, J. Yang, and Y.-Y. Lu, "Solution algorithms for singlemachine group scheduling with ready times and deteriorating jobs," Engineering Optimization, vol. 51, no. 5, pp. 862-874, 2019.

[10] X. Huang, "Bicriterion scheduling with group technology and deterioration effect," Journal of Applied Mathematics and Computing, vol. 60, no. 1-2, pp. 455-464, 2019.

[11] J.-B. Wang and X.-X. Liang, "Group scheduling with deteriorating jobs and allotted resource under limited resource availability constraint," Engineering Optimization, vol. 51, no. 2, pp. 231-246, 2019.

[12] X.-X. Liang, M. Liu, Y.-B. Feng, J.-B. Wang, and L.-S. Wen, "Solution algorithms for single-machine resource allocation scheduling with deteriorating jobs and group technology," Engineering Optimization, vol. 52, no. 7, pp. 1184-1197, 2020.

[13] H. Qin, Z.-H. Zhang, and D. Bai, "Permutation flowshop group scheduling with position-based learning effect," Computers \& Industrial Engineering, vol. 92, pp. 1-15, 2016.

[14] Y. Yin, M. Liu, T. C. E. Cheng, C.-C. Wu, and S.-R. Cheng, "Four single-machine scheduling problems involving due date determination decisions," Information Sciences, vol. 251, pp. 164-181, 2013. 
[15] D.-J. Wang, Y. Yin, J. Xu, W.-H. Wu, S.-R. Cheng, and C.-C. $\mathrm{Wu}$, "Some due date determination scheduling problems with two agents on a single machine," International Journal of Production Economics, vol. 168, pp. 81-90, 2015.

[16] D. Shabtay, "Optimal restricted due date assignment in scheduling," European Journal of Operational Research, vol. 252, no. 1, pp. 79-89, 2016.

[17] S. Li, C. T. Ng, and J. Yuan, "Group scheduling and due date assignment on a single machine," International Journal of Production Economics, vol. 130, no. 2, pp. 230-235, 2011.

[18] L. Sun, A. J. Yu, and B. Wu, "Single machine common flow allowance group scheduling with learning effect and resource allocation," Computers \& Industrial Engineering, vol. 139, Article ID 106126, 2020.

[19] D.-Y. Lv, S.-W. Luo, J. Xue, J.-X. Xu, and J.-B. Wang, “A note on single machine common flow allowance group scheduling with learning effect and resource allocation," Computers \& Industrial Engineering, vol. 151, Article ID 106941, 2021.

[20] W. Wang, J.-J. Wang, and J.-B. Wang, "Solution algorithms for single-machine group scheduling with learning effect and convex resource allocation," Complexity, vol. 2021, Article ID 6615824, 13 pages, 2021.

[21] L.-Y. Wang, M. Liu, J.-B. Wang, Y.-Y. Lu, and W.-W. Liu, "Optimization for due-date assignment single-machine scheduling under group technology," Complexity, vol. 2021, Article ID 6656261, 9 pages, 2021.

[22] A. Janiak, W. A. Janiak, T. Krysiak, and T. Kwiatkowski, "A survey on scheduling problems with due windows," European Journal of Operational Research, vol. 242, no. 2, pp. 347-357, 2015.

[23] G. A. Rolim and M. S. Nagano, "Structural properties and algorithms for earliness and tardiness scheduling against common due dates and windows: a review," Computers \& Industrial Engineering, vol. 149, Article ID 106803, 2020.

[24] J.-B. Wang, B. Zhang, L. Li, D. Bai, and Y.-B. Feng, "Duewindow assignment scheduling problems with position-dependent weights on a single machine," Engineering Optimization, vol. 52, no. 2, pp. 185-193, 2020.

[25] L.-Y. Wang, M. Liu, J.-B. Wang, Y.-Y. Lu, and W.-W. Liu, "Optimization for due-window assignment scheduling with position-dependent weights," Discrete Dynamics in Nature and Society, vol. 2020, Article ID 9746538, 7 pages, 2020.

[26] J.-B. Wang, Y. Hu, and B. Zhang, "Common due-window assignment for single-machine scheduling with generalized earliness/tardiness penalties and a rate-modifying activity," Engineering Optimization, vol. 53, no. 3, pp. 496-512, 2021.

[27] D.-Y. Lv and J.-B. Wang, "Study on resource-dependent nowait flow shop scheduling with different due-window assignment and learning effects," Asia Pacific Journal of Operational Research, vol. 16, Article ID 2150008, 2021.

[28] M. Ji, K. Chen, J. Ge, and T. C. E. Cheng, "Group scheduling and job-dependent due window assignment based on a common flow allowance," Computers \& Industrial Engineering, vol. 68, pp. 35-41, 2014.

[29] W.-X. Li and C.-L. Zhao, "Single machine scheduling problem with multiple due windows assignment in a group technology," Journal of Applied Mathematics and Computing, vol. 48, no. 1-2, pp. 477-494, 2015.

[30] X. Sun, X.-N. Geng, and T. Liu, "Due-window assignment scheduling in the proportionate flow shop setting," Annals of Operations Research, vol. 292, no. 1, pp. 113-131, 2020.

[31] J.-B. Wang, B. Cui, P. Ji, and W.-W. Liu, "Research on singlemachine scheduling with position-dependent weights and past-sequence-dependent delivery times," Journal of Combinatorial Optimization, vol. 41, no. 2, pp. 290-303, 2021.

[32] R. L. Graham, E. L. Lawler, J. K. Lenstra, and A. H. G. R. Kan, "Optimization and approximation in deterministic sequencing and scheduling: a survey," Annals of Discrete Mathematics, vol. 5, pp. 287-326, 1979.

[33] G. H. Hardy, J. E. Littlewood, and G. Polya, Inequalities, Cambridge University Press, Cambridge, UK, 2nd ed edition, 1967.

[34] C.-C. Wu, D. Bai, X. Zhang et al., "A robust customer order scheduling problem along with scenario-dependent component processing times and due dates," Journal of Manufacturing Systems, vol. 58, pp. 291-305, 2021.

[35] C.-C. Wu, D. Bai, J.-H. Chen et al., "Several variants of simulated annealing hyper-heuristic for a single-machine scheduling with two-scenario-based dependent processing times," Swarm and Evolutionary Computation, vol. 60, Article ID 100765, 2021.

[36] C.-C. Wu, J. N. D. Gupta, S.-R. Cheng, B. M. T. Lin, S.-H. Yip, and W.-C. Lin, "Robust scheduling for a two-stage assembly shop with scenario-dependent processing times," International Journal of Production Research, vol. 8, pp. 1-16, 2020.

[37] J. B. Wang, D. Y. Lv, J. Xu, P. Ji, and F. Li, "Bicriterion scheduling with truncated learning effects and convex controllable processing times," International Transactions in Operational Research, vol. 28, no. 3, pp. 1573-1593, 2021.

[38] J.-B. Wang, B. Zhang, and H. He, "A unified analysis for scheduling problems with variable processing times," Journal of Industrial and Management Optimization, vol. 20, 2021. 